\title{
Adaptação e Validação da Utrecht Work Engagement Scale (UWES) aplicada a Assistentes Sociais em Portugal
}

\author{
Adaptation and Validation of the Utrecht Work Engagement Scale (UWES) applied to Social Workers in Portugal
}

Artigo Original | Original Article

Helena Teles, PhD (1a), Nélson Ramalho, PhD Student (2b), Vanda Ramalho, PhD Student (3b), Sónia Ribeiro, PhD (4c)

(1) Centro de Administração e Políticas Públicas ISCSP

(2) Centro de Investigação e Estudos em Sociologia ISCTE-IUL;

(3) Centro Lusíada de Investigação em Serviço Social e Intervenção Social ULL-ISSS;

(4) Universidade Lusófona do Porto;

(a) Elaboração de análise estatística

(b) Elaboração do trabalho

(c) Contribuiu significativamente para a revisão e discussão do trabalho

Autor para correspondência: Nelson Ramalho, nelson_alves_ramalho@iscte-iul.pt

\section{Palavras-Chave}

Engagement

UWES

Assistentes Sociais

Portugal

\section{Keywords}

Engagement

UWES

Social Workers

Portugal

\section{RESUMO}

Objetivo: O presente estudo visa avaliar os itens e as respetivas dimensões da Utrecht Work Engagement Scale (UWES-17) de Schaufeli e Bakker (2009), aplicada a assistentes sociais a exercer funções em Portugal.

Método: Foi aplicada a versão portuguesa da UWES a uma amostra constituída por 1369 assistentes sociais portugueses, $94 \%$ do sexo feminino e $6 \%$ do sexo masculino, com uma média de idades de 39 anos (desviopadrão $=8,99)$. A habilitação académica mais frequente é a licenciatura $(63,8 \%)$ e no que respeita à atividade profissional possuíam, em termos médios, 12,99 anos de experiência (desvio-padrão = 8,28). A fidedignidade da escala foi avaliada através do coeficiente de alfa de Chronbach e a validade através da análise fatorial exploratória. Foi utilizada a pesquisa metodológica de natureza quantitativa.

Resultados: Os resultados alcançados vão ao encontro dos valores presentes em estudos anteriores quanto à sua consistência interna, tanto dos 17 itens que compõe a escala como das suas três dimensões ("vigor", “dedicação" e "absorção"). A UWES-17 neste estudo apresenta uma estrutura fatorial de três fatores, tal como o estudo original, mas a constituição dos itens de cada dimensão é diferente.

Conclusões: A escala UWES-17 apresenta-se com boas características psicométricas e uma boa consistência interna.

\section{ABSTRACT}

Objective: The present study aims to evaluate the items, and respective dimensions, of the Utrecht Work Engagement Scale (UWES-17) of Schaufeli e Bakker (2009), when applied to social workers exercising their profession in Portugal (UWES-17).

Method: The Portuguese version of the UWES was applied to a sample of 1369 Portuguese social workers, $94 \%$ of which female and $6 \%$ male, with an average age of 39 years (standard deviation $=8,99$ ). The most frequent academic qualification is graduation (63,8\%) and in terms of professional activity they had, in average terms, 12,99 years of experience (standard deviation $=8,28$ ). The reliability of the scale was assessed using the Chronbach alpha coefficient and the validity using the exploratory factorial analysis. Quantitative methodological research was used.

Results: The results obtained agree with the values presented in previous studies regarding their internal consistency, both of the 17 items that make up the scale and of its three dimensions ("force", "dedication" and "absorption"). The UWES-17 in this study presents a factorial structure of three factors, just like the original study, but the constitution of the items of each dimension is different. The UWES-17 scale presents good psychometric characteristics and good internal consistency.

Conclusions: The UWES-17 scale presents good psychometric characteristics and good internal consistency. 


\section{INTRODUÇÃO}

A psicologia, ao longo da sua história, deu mais primazia ao estudo dos aspetos negativos do ser humano em geral (problemas, sintomas e doenças) do que aos positivos (Bakker et al., 2008). Como resposta a esta tendência, surge a chamada "Psicologia Positiva", que foca os aspetos psicológicos positivos. É a partir desta corrente, que na década de 90 Kahn começa a desenvolver os primeiros estudos sobre engagement, como consequência às inúmeras pesquisas sobre os problemas, sintomas e doenças laborais, como o burnout.

$O$ engagement ${ }^{1}$ no trabalho, definido enquanto um estado mental positivo e de bem-estar relacionado com o contexto laboral, tende a ser visto como oposto ao burnout. Contrariamente aos trabalhadores que sofrem desta síndrome - manifestado em estado de exaustão emocional, despersonalização e reduzida realização pessoal (Maslach e Leiter, 1997) - os que se encontram envolvidos na sua atividade profissional revelam energia, eficácia e capacidade para lidar positivamente com os desafios do trabalho.

Porém, se para Maslach e Leiter (1997), o engagement e o burnout, enquanto estados psicológicos do bem-estar laboral (um possuindo qualidades positivas e outro negativas), encontram-se negativamente correlacionados, para Schaufeli e Bakker (2003) esta correlação nem sempre acontece. Por exemplo, quando um trabalhador não está afetado pelo síndrome de burnout, tal não significa que apresente nível de engagement no seu posto de trabalho. E o contrário, também é verdade, ou seja, quando um trabalhador possui baixos níveis de engagement, não significa que ele esteja em situação de burnout. Teoricamente, estes dois conceitos não podem ser estudados em simultâneo, dado apresentarem-se como distintos e independentes um do outro. No entanto, na prática, considera-se haver uma possível correlação entre o burnout e o engagement. Existem evidências empíricas que apresentam correlação negativa do engagement com o burnout (Salanova, Schaufeli, Llorens, Peiró, e Grau, 2000; Durán, Extremera, e Rey, 2005) e correlação positiva com o desenvolvimento profissional (Martínez e Salanova, 2003), com as competências emocionais, o bem-estar emocional e a felicidade (Extremera, Durán, e Rey, 2005).

Schaufeli, Salanova, González-Romá e Bakker (2002, p. 74) definem o engagement como um estado afetivocognitivo positivo, persistente e abrangente (não sendo característico de um evento, indivíduo ou comportamento momentâneo), relacionado com o mundo laboral, constituído por três dimensões: comportamental ("vigor"), emocional ("dedicação") e cognitiva ("absorção"). O "vigor" caracteriza-se por altos níveis de energia, resiliência mental, vontade de investir e persistir face a dificuldades no trabalho; a "dedicação" pelo forte envolvimento, entusiasmo, orgulho, desafio, inspiração no desempenho da atividade profissional e atribuição de significado ao trabalho desenvolvido; e a "absorção" pela "imersão" e concentração total nas atividades em que o indivíduo se encontra implicado, de tal forma que possui dificuldade em desapegar-se do seu trabalho, dando a sensação de o tempo passar rapidamente. Estar absorvido no trabalho consiste, pois, num estado de atenção focalizada e satisfação intrínseca (Csikszentmihalyi, 1990). $\mathrm{O}$ engagement profissional caracteriza-se, pois, por uma forte identificação e altos níveis de energia despendidos no trabalho.

Dado a falta de instrumentos para se avaliar e medir o engagement, os autores Schaufeli e Bakker (2009) elaboraram o questionário Utrecht Work Engagement Scale (UWES). Originalmente, este instrumento, construído em 1999, era composto por 24 itens, dos quais nove eram relativos ao "vigor" e oito à "dedicação". Mais tarde, em 2002, após uma avaliação psicométrica, sete desses itens demonstraram-se inconsistentes, tendo sido eliminados. A versão UWES de 17 itens é, assim, composta por seis itens de "vigor", cinco de "dedicação" e seis de "absorção" (Schaufeli et al., 2002).2

Entre 1999 e 2003, a escala UWES-17 foi sujeita a vários testes psicométricos preliminares. Num primeiro momento, aplicada a uma grande amostra de trabalhadores $(n=9679)$ pertencentes a diferentes grupos profissionais na Holanda e Bélgica; e, mais tarde, estendida a outros países. Estes testes tinham a pretensão de avaliar a distribuição dos itens, a consistência interna das subescalas, a estrutura fatorial da UWES, a relação com diferentes variáveis (o burnout, a idade e o sexo), bem como as diferenças entre os grupos profissionais e entre países. Dos seus resultados, Schaufeli e Bakker (2009), apresentam, para este instrumento, propriedades psicométricas muito satisfatórias. A consistência interna revelou-se alta, tal como a estabilidade temporal. A estrutura fatorial foi confirmada, revelando níveis altos de correlação entre os fatores que a compõem, não variável entre amostras de diversos países. No que se refere ao burnout, observou-se uma correlação negativa com o engagement. Foram encontradas pequenas diferenças estatisticamente significativas nos níveis de engagement relativas a algumas variáveis, como a idade, o sexo e os

\footnotetext{
1 O conceito de engagement é relativamente recente. É da autoria de Kahn que, em 1990, o cunhou no artigo Psychological conditions of personal engagement and disengagement at work. Até o momento ainda não existe, em português, um termo que abranja a natureza do conceito e que permita traduzi-lo de forma clara. Por isso, uma aproximação possível seria "envolvimento".
}

2 Atualmente, existe, também uma versão de UWES- 15 e UWES-9 itens. 
grupos profissionais, mas com pouca relevância a nível prático. Deste modo, os autores consideram a UWES um instrumento válido e adequado para ser usado em investigações futuras com outros grupos profissionais, podendo estes estarem integrados em diferentes territórios ou países.

O presente estudo pretende analisar, por um lado, as dimensões do "vigor", "dedicação" e "absorção" como formas específicas de engagement (Schaufeli e Bakker, 2003). Dado que vários estudos nacionais e internacionais têm observado elevados riscos de burnout presentes na população dos assistentes sociais (Abdallah, 2009; Carrera, 2011; Collins, 2008; Davies, 1998; Hamama, 2012; Kim, 2011; Lloyd, King e Chenoweth, 2002; Lonne, 2003; Ramiro, 2014; Takeda, Yokoyama, Miyake, e Ohida, 2002) e de outros trabalhadores sociais (Aritzeta e Gartzia, 2009; Extremera et al., 2005; Fong e Ng, 2012) devido aos contextos profissionais permeáveis a situações de stress, pretendese, por outro lado, complementar estes estudos, avaliando as características psicométricas da UWES quando aplicada a esta classe profissional, em Portugal.

A UWES é, atualmente, o instrumento mais utilizado em estudos internacionais para avaliar o engagement profissional. Ter uma ferramenta de medição válida e padronizada do constructo de engagement é essencial para facilitar uma melhor compreensão do envolvimento, da regulação das emoções e do bem-estar dos trabalhadores nos contextos laborais, razão pela qual, se pretendeu validar a escala UWES-17 para a população de assistentes sociais em Portugal (UWES-17) dada a sua inexistência, permitindo melhorar e prevenir os efeitos negativos dos riscos psicossociais em contextos sócio comunitários. Em Portugal já existe um estudo relativo à validação desta escala, mas respeitante aos profissionais dos cuidados de saúde primários (Martins, 2013).

\section{MÉTODO}

De acordo com a finalidade e objetivos deste trabalho realizou-se um estudo metodológico de natureza quantitativa do tipo descritivo-correlacional que permite obtenção, organização e análise de dados, através da elaboração, validação e avaliação do instrumento e técnicas de pesquisa.

\section{Instrumentos}

Para avaliar o engagement nos assistentes sociais utilizou-se a UWES-17 de Schaufeli e Bakker (2009), na versão traduzida para o português (Angst, Benevides-
Pereira, e Porto-Martins, 2009), adaptando-se, no entanto, algumas expressões brasileiras ao português europeu e promovendo-se a linguagem paritária3, conforme Tabela 1. Esta escala - que pretende avaliar em que medida os assistentes sociais se encontram envolvidos com o seu trabalho (e não a eficácia do trabalho executado) encontra-se estruturada por dezassete itens, divididos em três dimensões relacionáveis entre si: o "vigor", com seis itens $(1,4,8,12,15,16)$, a "dedicação" com cinco $(2,5,7$, 10, 13) e a "absorção" com seis (3, 6, 9, 11, 14,16). Os profissionais responderam a cada um dos itens de acordo com uma escala ordinal do tipo Likert com sete alternativas de resposta, composta por valores variáveis entre 0 (se nunca teve esse sentimento ou crença) e 6 (se o sentiam ou tinham frequentemente).

\section{Tabela 1}

Escala UWES-17 Aplicada aos Assistentes Sociais Portugueses

\section{Itens da UWES-17}

1. No meu trabalho sinto-me chei@ de energia

2. Acho que o meu trabalho tem muito significado e utilidade

3. O tempo passa a voar quando estou a trabalhar

4. No meu trabalho sinto-me com força e energia

5. Estou entusiasmad@ com o meu trabalho

6. Quando estou a trabalhar esqueço tudo o que se passa à minha "volta"

7. O meu trabalho inspira-me

8. Quando me levanto de manhã apetece-me ir trabalhar

9. Sinto-me feliz quando estou a trabalhar intensamente

10. Estou orgulhos@ do que faço neste trabalho

11. Estou imers@ no meu trabalho

12. Sou capaz de ficar a trabalhar por períodos de tempo muito longos

13. O meu trabalho é desafiante para mim

14. "Deixo-me ir" quando estou a trabalhar

15. Sou uma pessoa com muita resistência mental no meu trabalho

16. É-me difícil desligar do meu trabalho

17. No meu trabalho sou sempre perseverante (não desisto), mesmo quando as coisas não estão a correr bem

\section{Procedimento}

A escala UWES-17 foi aplicada aos assistentes sociais portugueses através da aplicação de um questionário, elaborado com recurso à plataforma Google Docs, disponível on-line por um período aproximado de dois meses (Setembro a Outubro de 2015). O questionário apresentava confidencialidade e garantias de anonimato das informações recolhidas. Para a sua divulgação recorreu-se à rede social Facebook e ao envio de 6.261 correios electrónicos para assistentes sociais, apelando à sua participação através de auto-preenchimento. No total foram obtidas 1434 respostas. Excluíram-se 65, dado não

\footnotetext{
3 Recorremos à utilização do símbolo “@” na aplicação da UWES-17 (passando, por exemplo, a afirmação: “No meu trabalho sinto-me cheio de energia” a estar constituída como “No meu trabalho sinto-me chei@ de energia”) a fim de garantir uma linguagem inclusiva que contemplasse não só as categorias de homem / mulher como as restantes que integram o espectro não-binário.
} 
Tabela 2

Caracterização da Amostra $(N=1369)$

\begin{tabular}{|c|c|c|c|c|c|}
\hline Características & $n$ & $\%$ & Características & $\mathbf{n}$ & $\%$ \\
\hline Sexo & & & Sector Laboral & & \\
\hline Feminino & 1287 & 94 & Público & 637 & 46,5 \\
\hline Masculino & 82 & 6 & Privado & 656 & 47,9 \\
\hline Grupo Etário & & & Ambos (público e privado) & 76 & 5,6 \\
\hline $20-29$ anos & 194 & 14,2 & Vencimento Bruto & & \\
\hline 30-39 anos & 587 & 42,9 & Sem rendimentos & 5 & $0,4^{*}$ \\
\hline $40-49$ anos & 397 & 29 & <S.M.N. & 17 & 1,2 \\
\hline $50-59$ anos & 162 & 11,9 & S.M.N. $(505 €)$ & 7 & 0,5 \\
\hline $60-69$ anos & 29 & 2 & $505 €$ a $600 €$ & 23 & 1,7 \\
\hline Habilitações Académicas & & & $600 €$ a $800 €$ & 120 & 8,8 \\
\hline Licenciatura & 874 & 63,8 & $800 €$ a $1.000 €$ & 263 & 19,2 \\
\hline Pós-Graduação & 294 & 21,5 & $1000 €$ a $1200 €$ & 271 & 19,8 \\
\hline Mestrado & 187 & 13,7 & $1200 €$ a $1500 €$ & 347 & 25,3 \\
\hline Doutoramento & 13 & 0,9 & $1500 €$ a $2000 €$ & 232 & 16,9 \\
\hline Pós-Doutoramento & 1 & 0,1 & $>2000 €$ & 84 & 6,1 \\
\hline Anos de Exercício Profissional & & & Satisfacão com o Vencimento & & \\
\hline$<10$ anos & 642 & 46,9 & $\operatorname{Sim}$ & 127 & 9,3 \\
\hline 11 a 20 anos & 519 & 37,9 & Não & 1018 & 74,4 \\
\hline 21 a 30 anos & 156 & 11,4 & Mais ou menos & 224 & 16,4 \\
\hline 31 a 40 anos & 47 & 3,4 & Avaliação do Ambiente de Trabalho & & \\
\hline$>41$ anos & 5 & 0,4 & Muito Bom & 210 & 15,3 \\
\hline Campo de Intervenção Profissional & & & Bom & 557 & 40,7 \\
\hline $3^{\mathrm{a}}$ Idade & 226 & 16,5 & Satisfatório & 481 & 35,1 \\
\hline Ação Social & 352 & 25,7 & Mau & 65 & 4,7 \\
\hline Crianças e Jovens & 197 & 14,4 & Muito Mau & 9 & 0,7 \\
\hline Educação/formação & 35 & 2,6 & Avaliação das Condições Materiais de Trabalho & & \\
\hline Empresas & 9 & 0,7 & Muito Boas & 125 & 9,1 \\
\hline Ensino e investigação & 9 & 0,7 & Boas & 419 & 30,6 \\
\hline Habitação & 20 & 1,5 & Satisfatórias & 597 & 43,6 \\
\hline Justiça & 12 & 0,9 & Más & 195 & 14,2 \\
\hline Saúde & 297 & 21,7 & Muito Más & 33 & 2,4 \\
\hline Deficiência e Reabilitação & 84 & 6,1 & & & \\
\hline Múltiplas áreas de Intervenção & 32 & 2,3 & & & \\
\hline
\end{tabular}

deterem os critérios definidos para o universo em análise4. Face ao exposto, o estudo totalizou 1369 respostas válidas. Os dados foram tratados com recurso ao Software Statistical Package for the Social Sciences (SPSS - versão 22 ), tendo como variáveis base os 17 itens da UWES. Dado que a validação de uma escala é sempre determinada pelo estudo da sua validade e fiabilidade, foram realizados testes de fiabilidade à consistência internas da escala, através do cálculo dos coeficientes Alfa de Cronbach, correlação de Pearson e teste $t$ de Student. Face à validade do constructo foi realizada a análise fatorial exploratória.

\section{Amostra}

O tamanho da amostra foi calculado através da fórmula para populações finitas e variável qualitativa, recorrendo-se a uma amostra aleatória simples (Sarmento, 2008, p. 25), que garante um nível de confiança e precisão aceitáveis. Assume-se um universo de 14875 que reflete, de acordo com Branco (2009), o número global de licenciados em serviço social, em Portugal6. O nível de respostas $(n=1369)$ foi acima da dimensão exigida estatisticamente. Calculou-se o nível de precisão, cuja margem de erro foi de, aproximadamente, 2,5\%.

A amostra do estudo (Tabela 2) foi constituída por assistentes sociais maioritariamente do sexo feminino (94\%), com idades compreendidas entre 22 e os 69 anos. A média etária situava-se nos 39 anos ( $D P=8,99$; Moda $=34$ ). A habilitação académica mais frequente era a licenciatura $(63,8 \%)$, sendo que $36,2 \%$ detinham uma pós-graduação, mestrado, doutoramento ou pós-doutoramento. No que respeita à atividade profissional possuíam, em termos médios, 12,99 anos de experiência ( $D P=8,28)$, encontrandose a exercer funções maioritariamente no domínio da ação social $(25,7 \%)$, saúde $(21,7 \%)$ ou gerontologia $(16,5 \%)$, distribuídos, de forma muito idêntica, pelo setor público $(46,5 \%)$ e privado $(47,9 \%)$. Em termos de vencimento bruto, $68,1 \%$

4 Nomeadamente, ao nível da licenciatura em serviço social/política social, a exercerem funções em Portugal.

5 A consistência interna representa a média das correlações entre todas as questões do questionário.

6 Embora os dados fossem apenas uma estimativa realizada no ano de 2009, estes eram, até ao momento, os únicos existentes a nível nacional. 


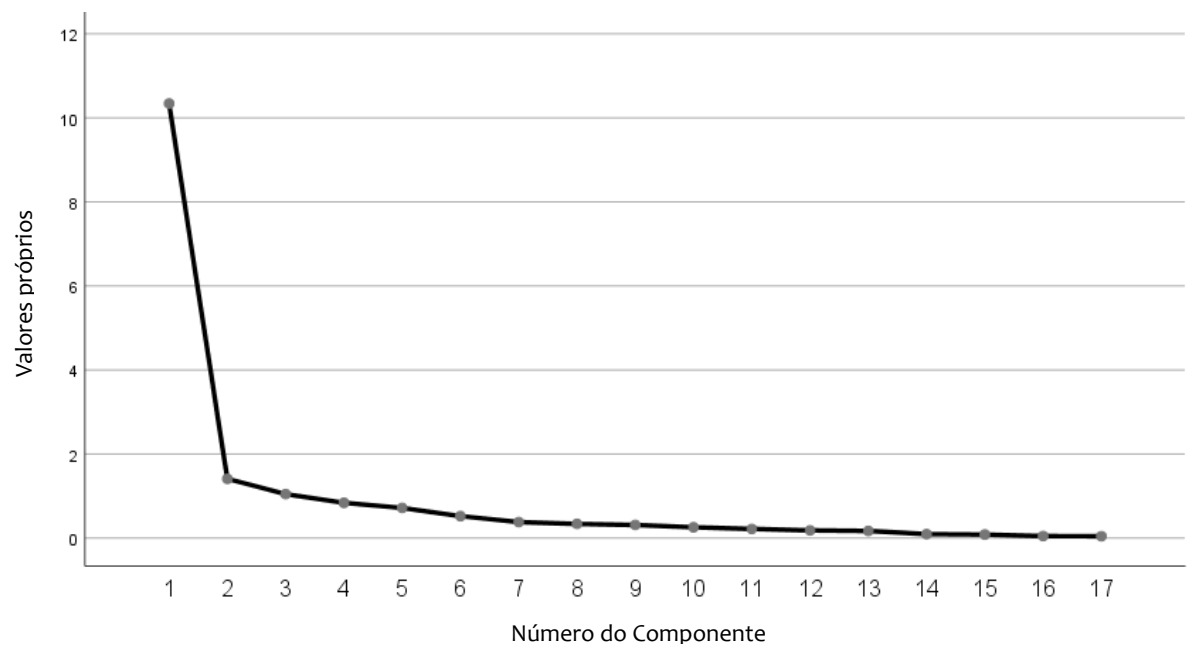

Figura 1. Scree Plot dos Componentes da UWES

auferia rendimentos mensais superiores a 1000 euros. Porém, 74,4\% revelava não estar satisfeita com o seu vencimento. $\mathrm{O}$ ambiente de trabalho foi avaliado como sendo "bom" $(40,7 \%)$ e as condições materiais disponibilizadas para o exercício das suas funções como "satisfatórias" (43,6\%).

\section{RESULTADOS}

\section{Validade}

De forma a averiguar se existiria a possibilidade de se proceder à análise das correlações do conjunto de itens, através da análise fatorial, estudámos o valor de medida de adequabilidade através do Kaiser-Meyer-Olkin Measure of Sampling Adequacy $(K M O) 7$ e do teste de Bartlett ${ }^{8}$. No estudo em presença, encontramos um KMO de 0,88 e no teste de Bartlett, um valor significativo inferior a 0,001. Assim, uma vez que ambos apresentam valores aceitáveis, existem condições para a realização de uma análise fatorial. Prosseguiu-se com a extração a partir dos 17 itens da Escala UWES, através da análise fatorial exploratória (Tabela 3 ).

Para determinar o número de componentes a incluir na análise utilizou-se o critério de Kaiser (exclui as componentes cujos valores próprios são inferiores a 1), cumprindo-se o critério de que os fatores devem explicar aproximadamente $70 \%$ da variação total observada nas variáveis originais. São retidos três fatores, que explicam
$75,3 \%$ da variação total observada nas 17 variáveis originais. De seguida apresenta-se o diagrama Scree Plot (Figura 1). Atendendo a que "os valores próprios representados em relação ao número de fatores a reter, são os que correspondem à maior inclinação de reta, ou seja, a um maior afastamento entre valores próprios" (Pestana e Gageiro, 2000, p. 399), o scree plot da fatorização corrobora os três fatores, o que é coincidente com a proposta dos autores.

A Tabela 4 apresenta a matriz dos componentes após rotação Varimax, salientando-se os fatores associados a cada uma das variáveis.

O Fator I, resultante da análise fatorial exploratória, integra todos os itens da dimensão "dedicação" (2, 5, 7, 10 e 13), mas integra também 3 itens do "vigor" $(1,4$ e 8$)$ e 3 itens da "absorção" (3, 6 e 11). O Fator II integra os itens 9, 15 e 17 , sendo que estes dois últimos pertencem à dimensão do "vigor" e o primeiro à dimensão de "absorção". Por fim, o Fator III integra os itens 12, 14 e 16, sendo o primeiro relativo à dimensão "vigor" e os restantes à dimensão "absorção". Para esta análise dos dados foi tido em consideração o defendido por Costello e Osborne (2005), designadamente o facto da constituição de cada fator ter um mínimo de 3 itens, um valor de saturação fatorial dos itens superior a 0,30 e a não existência de saturações duplas. Os resultados corroboram a estrutura fatorial proposta pelos autores da escala, com três dimensões, não se corroborando o agrupamento de itens das dimensões.

\footnotetext{
7 “O KMO perto de 1 indica coeficientes de correlação parciais pequenos, enquanto que valores próximos de zero indica que a análise fatorial pode não ser uma boa ideia, porque existe uma correlação fraca entre as variáveis” (Pestana e Gageiro, 2000, p. 397). O KMO compara as correlações simples com as correlações parciais observadas entre as mesmas. Os valores de KMO podem variar de o a 1, sendo que a partir de 0,7, se considera aceitável o valor para analise fatorial; de 0,5 a 0,7 é considerado aceitável; de 0,7 a 0,8 é considerado bom; de 0,8 a 0,9 é ótimo; e acima de 0,9 é considerado soberbo (Malheiro, 2010).

8 Neste teste, sempre que o valor do Qui-quadrado é significativo (< 0,001), as correlações entre as variáveis são adequadas para a análise fatorial. Este
} “testa a hipótese de a matriz das correlações ser a matriz de identidade, cujo determinante é igual a 1.” (Pestana e Gageiro, 2000, p. 251). 
Tabela 3

Variação Total da Análise de Componentes Principais

\begin{tabular}{|c|c|c|c|c|c|c|}
\hline \multirow{2}{*}{ Componente } & \multicolumn{3}{|c|}{ Valores próprios iniciais } & \multicolumn{3}{|c|}{ Soma de extração dos pesos fatoriais ao quadrado } \\
\hline & Total & \% da Variância & Cumulativo \% & Total & \% da Variância & Cumulativo $\%$ \\
\hline 1 & 10,341 & 60,8 & 60,8 & 10,341 & 60,8 & 60,8 \\
\hline 2 & 1,410 & 8,3 & 69,1 & 1,410 & 8,3 & 69,1 \\
\hline 3 & 1,046 & 6,2 & 75,3 & 1,046 & 6,2 & 75,3 \\
\hline 4 & 0,838 & 4,9 & 80,2 & & & \\
\hline 5 & 0,717 & 4,3 & 84,4 & & & \\
\hline 6 & 0,522 & 3,1 & 87,5 & & & \\
\hline 7 & 0,381 & 2,2 & 89,7 & & & \\
\hline 8 & 0,337 & 2,0 & 91,7 & & & \\
\hline 9 & 0,312 & 1,8 & 93,6 & & & \\
\hline 10 & 0,256 & 1,5 & 95,1 & & & \\
\hline 11 & 0,218 & 1,3 & 96,4 & & & \\
\hline 12 & 0,182 & 1,1 & 97,4 & & & \\
\hline 13 & 0,170 & 1,0 & 98,4 & & & \\
\hline 14 & 0,093 & 0,5 & 99,0 & & & \\
\hline 15 & 0,083 & 0,5 & 99,5 & & & \\
\hline 16 & 0,048 & 0,3 & 99,7 & & & \\
\hline 17 & 0,044 & 0,3 & 100,0 & & & \\
\hline
\end{tabular}

Nota. Método de Extração: Análise de Componentes Principais.

Tabela 4

Rotação Varimax de Componentes Principais da PSS

\begin{tabular}{|c|c|c|c|}
\hline \multicolumn{4}{|l|}{ Matriz de Componentesa } \\
\hline & \multicolumn{3}{|c|}{ Componente } \\
\hline & 1 & 2 & 3 \\
\hline 1. No meu trabalho sinto-me chei@ de energia & 0,853 & 0,145 & 0,217 \\
\hline 2. Acho que o meu trabalho tem muito significado e utilidade & 0,615 & 0,522 & 0,090 \\
\hline 3. O tempo passa a voar quando estou a trabalhar & 0,821 & 0,235 & 0,185 \\
\hline 4. No meu trabalho sinto-me com força e energia & 0,920 & 0,218 & 0,132 \\
\hline 5. Estou entusiasmad@ com o meu trabalho & 0,893 & 0,310 & 0,127 \\
\hline 6. Quando estou a trabalhar esqueço tudo o que se passa à minha "volta" & 0,488 & 0,256 & 0,380 \\
\hline 7. O meu trabalho inspira-me & 0,766 & 0,393 & 0,254 \\
\hline 8. Quando me levanto de manhã apetece-me ir trabalhar & 0,848 & 0,273 & 0,172 \\
\hline 9. Sinto-me feliz quando estou a trabalhar intensamente & 0,430 & 0,651 & 0,014 \\
\hline 10. Estou orgulhos@ do que faço neste trabalho & 0,618 & 0,562 & 0,099 \\
\hline 11. Estou imers@ no meu trabalho & 0,598 & 0,541 & 0,259 \\
\hline 12. Sou capaz de ficar a trabalhar por períodos de tempo muito longos & 0,445 & 0,363 & 0,640 \\
\hline 13. O meu trabalho é desafiante para mim & 0,704 & 0,279 & 0,437 \\
\hline 14. “Deixo-me ir" quando estou a trabalhar & 0,561 & 0,364 & 0,637 \\
\hline 15. Sou uma pessoa com muita resistência mental no meu trabalho & 0,170 & 0,808 & 0,256 \\
\hline 16. É-me difícil desligar do meu trabalho & 0,031 & 0,088 & 0,832 \\
\hline 17. No meu trabalho sou sempre perseverante (não desisto), mesmo quando as coisas não estão a correr bem & 0,179 & 0,850 & 0,301 \\
\hline
\end{tabular}

Nota. aA rotação convergiu em 3 iterações. Método de extração: Análise de Componentes Principais. Método de rotação: Varimax com Normalização Kaiser.

\section{Fiabilidade}

A fim de procurar validar a adaptação da UWES-17 à população de assistentes sociais portugueses, realizou-se uma análise das características psicométricas da escala, tendo-se testado a sua consistência interna (fiabilidade) através do Alfa de Cronbach, que pondera o rácio existente entre a variância total dos 17 itens que compõem a UWES-17 e a variância de cada um dos itens. Autores vários (Davis, 1964; Kaplan e Saccuzzo, 1982; Murphy e Davidsholder, 1988; Nunnally, 1978) têm definido critérios de recomendação de fiabilidade estimada para o Alfa de Cronbach, estimando intervalos considerados aceitáveis para a utilização de escalas científicas (Tabela 5). 
Tabela 5

Critérios de Recomendação de Fiabilidade Estimada para o Alfa de Cronbach

\begin{tabular}{|c|c|c|}
\hline Autor & Condição & $\begin{array}{c}\text { Alfa considerado } \\
\text { aceitável }\end{array}$ \\
\hline \multirow{2}{*}{$\begin{array}{l}\text { Davis } \\
\text { (2008, p. 24) }\end{array}$} & Previsão individual & Acima de 0,75 \\
\hline & Previsão para grupos de $25-50$ & Acima de 0,5 \\
\hline \multirow{2}{*}{$\begin{array}{l}\text { Kaplan e Saccuzzo } \\
(1982, \text { p. 106) }\end{array}$} & Investigação fundamental & $0,7-0,8$ \\
\hline & Investigação aplicada & 0,95 \\
\hline \multirow{4}{*}{$\begin{array}{l}\text { Murphy e } \\
\text { Davidsholder (1988, } \\
\text { p. 89) }\end{array}$} & Fiabilidade inaceitável & $<0,6$ \\
\hline & Fiabilidade baixa & 0,7 \\
\hline & Fiabilidade moderada a elevada & $0,8-0,9$ \\
\hline & Fiabilidade elevada & $>0,9$ \\
\hline \multirow{3}{*}{$\begin{array}{l}\text { Nunnally } \\
(1978 \text {, p. 245-246) }\end{array}$} & Investigação preliminar & 0,7 \\
\hline & Investigação fundamental & 0,8 \\
\hline & Investigação aplicada & $0,9-0,95$ \\
\hline
\end{tabular}

Nota. Adaptado de Peterson (1994).

Os autores responsáveis pelos estudos relativos à UWES-17 (Demerouti, Bakker, Janssen, e Schaufeli, 2001; Montgomery, Peeters, Schaufeli e Den Ouden, 2003; Salanova et al., 2000; Salanova, Brescó e Schaufeli, 2005; Salanova, Carrero, Pinanzo e Schaufeli, 2003; Salanova, Grau, Llorens e Schaufeli, 2001; Schaufeli e Bakker, 2004) determinaram a sua consistência interna dentro do intervalo 0,91-0,96 (Schaufeli e Bakker, 2009, p. 13).

Dos resultados da aplicação do Alfa de Cronbach à UWES-17 constata-se que esta escala apresenta um bom coeficiente de consistência interna, com um valor de 0,95. O valor mínimo do Alfa de Cronbach $(0,95)$ foi verificado no item “5. Estou entusiasmad@ com o meu trabalho" e o mais elevado $(0,96)$ no item "16. É-me difícil desligar do meu trabalho", conforme Tabela 6. Este valor revela, pois, que estamos perante um instrumento cujo grau de consistência e fiabilidade interna é adequado dado encontrar-se dentro dos limites estabelecidos pelos autores.

No que concerne ao Alfa de Cronbach das três dimensões ("vigor", “dedicação" e "absorção"), os valores apurados na UWES-17 aplicada a assistentes sociais são superiores à UWES-17 (Tabela 7). A dimensão na qual se verifica um Alfa de Cronbach substancialmente superior é na "absorção" na qual a UWES-17 apresenta um Alfa de Cronbach de 0,83 e na UWES-17 aplicada a assistentes sociais um valor de 0,92, acima do intervalo enunciado para a UWES-17 estabelecido entre 0,79-0,88 (Schaufeli e Bakker, 2009, p. 21). Deste modo, considerase que os dados são aceitáveis como unidimensionais em cada uma das dimensões, apresentando todas elas um nível de consistência adequado na amostra em estudo.

Tabela 7

Alfa de Cronbach das Escalas do UWES-17 vs. UWES-17 Aplicada a Assistentes Sociais

\begin{tabular}{lcc}
\hline & $\begin{array}{c}\text { UWES-17 } \\
(N=12 ~ 161) *\end{array}$ & $\begin{array}{c}\text { UWES-17 no presente estudo } \\
(N=1369)\end{array}$ \\
\hline Vigor & 0,82 & 0,89 \\
Dedicação & 0,89 & 0,91 \\
Absorção & 0,83 & 0,92 \\
Total & 0,93 & 0,94 \\
\hline
\end{tabular}

Nota. *adaptado de Schaufeli e Bakker (2009, p. 21).

Tabela 6

Estatística Descritiva e Alfa de Cronbach

\begin{tabular}{|c|c|c|c|c|c|c|}
\hline & Média & Desvio Padrão & $\begin{array}{l}\text { Média da escala se o } \\
\text { item for excluído }\end{array}$ & $\begin{array}{l}\text { Variância da escala se } \\
\text { o item for excluído }\end{array}$ & $\begin{array}{l}\text { Correlação item- } \\
\text { total corrigida }\end{array}$ & $\begin{array}{c}\text { Alfa de Cronbach se o } \\
\text { item for excluído }\end{array}$ \\
\hline UWES-APS 1 & 4,13 & 1,48 & 66,05 & 400,56 & 0,73 & 0,95 \\
\hline UWES-APS 2 & 4,98 & 1,38 & 65,21 & 406,50 & 0,68 & 0,95 \\
\hline UWES-APS 3 & 4,65 & 1,53 & 65,53 & 402,73 & 0,67 & 0,95 \\
\hline UWES-APS 4 & 4,11 & 1,54 & 66,08 & 394,47 & 0,81 & 0,95 \\
\hline UWES-APS 5 & 3,97 & 1,64 & 66,21 & 389,08 & 0,84 & 0,95 \\
\hline UWES-APS 6 & 3,78 & 1,81 & 66,4 & 395,66 & 0,66 & 0,95 \\
\hline UWES-APS 7 & 3,82 & 1,75 & 66,37 & 386,85 & 0,81 & 0,95 \\
\hline UWES-APS 8 & 3,65 & 1,89 & 66,54 & 383,93 & 0,79 & 0,95 \\
\hline UWES-APS 9 & 4,08 & 1,69 & 66,1 & 395,51 & 0,71 & 0,95 \\
\hline UWES-APS 10 & 4,44 & 1,60 & 65,74 & 393,43 & 0,79 & 0,95 \\
\hline UWES-APS 11 & 4,19 & 1,59 & 66,00 & 394,78 & 0,77 & 0,95 \\
\hline UWES-APS 12 & 4,02 & 1,70 & 66,17 & 397,25 & 0,68 & 0,95 \\
\hline UWES-APS 13 & 4,03 & 1,80 & 66,16 & 385,56 & 0,81 & 0,95 \\
\hline UWES-APS 14 & 3,94 & 1,72 & 66,24 & 390,22 & 0,78 & 0,95 \\
\hline UWES-APS 15 & 4,17 & 1,58 & 66,01 & 402,59 & 0,65 & 0,95 \\
\hline UWES-APS 16 & 3,71 & 1,84 & 66,47 & 413,44 & 0,39 & 0,96 \\
\hline UWES-APS 17 & 4,53 & 1,51 & 65,66 & 404,69 & 0,64 & 0,95 \\
\hline
\end{tabular}


Recorreu-se ao teste $t$ de Student para confirmar a significância das diferenças entre dimensões. $\mathrm{Na}$ Tabela 8 são apresentados os valores da média, do erro padrão e do desvio padrão dos resultados obtidos na UWES-17 aplicada a assistentes sociais em comparação com os dados apresentados pela UWES-17. As médias observadas nas três dimensões encontram-se acima de 4 pontos ("uma vez por semana"), numa escala de o ("nunca") até 6 ("todos os dias"), sendo o ponto médio 3 ("algumas vezes por mês"). O nível total do engagement quer da UWES-17 como da UWES-17 aplicada a assistentes sociais estão acima do ponto médio (3), revelando valores semelhantes entre si.

Além da observação da média, desvio e erro padrão, realizou-se a comparação das percentagens de scores9. Constatou-se (Tabela 9) que tanto no score total da UWES-17 como da UWES-17 aplicada a assistentes sociais, mais de metade dos trabalhadores detinham um score principal de 5 ou 6: 59,1\% (UWES-17) e $61,2 \%$ (UWES-17 aplicada a assistentes sociais) dos trabalhadores selecionaram as respostas "algumas vezes por semana" (5) ou "todos os dias" (6). Relativamente às opções "algumas vezes por ano" (1) e "uma vez ou menos por mês" (2) quer a UWES-17 quer a UWES-17 aplicada no nosso estudo apresentaram valores residuais de $4,2 \%$ e 7,6\% respetivamente. Tais resultados evidenciam não existirem diferenças substanciais nos scores de engagement entre escalas.

Após a verificação da distribuição dos scores em percentagens, efetuou-se uma análise aos indicadores estatísticos da média, desvio e erro padrão dos scores normativos para as três dimensões. Também aqui não se verificaram discrepâncias expressivas entre os valores da UWES-17 e da UWES-17 no presente estudo (Tabela 10). Destaca-se, no entanto, os valores da média da UWES-17 no presente estudo ligeiramente superiores. Contudo, estes não comprometem a adequação das características psicométricas da escala aplicada.

Para a fixação de normas estatísticas standard para a UWES-17, os autores (Schaufeli e Bakker, 2009) elencaram um conjunto de cinco categorias: "muito baixo", "baixo", "médio", "alto" e "muito alto". A Tabela 11 mostra a definição e atribuição de intervalos para estas cinco categorias. Esta classificação foi motivada, entre outras características psicométricas, pela distribuição dos itens e considerando a preocupação com a mensuração do erro padrão.

Tabela 8

Média, Erro Padrão e Desvio Padrão das Dimensões do UWES-17 e da UWES-17 Aplicada a Assistentes Sociais

\begin{tabular}{|c|c|c|c|c|c|c|}
\hline & \multicolumn{3}{|c|}{$\begin{array}{c}\text { UWES-17 } \\
(N=12161)\end{array}$} & \multicolumn{3}{|c|}{$\begin{array}{l}\text { UWES-17 no presente estudo } \\
\qquad(N=1369)\end{array}$} \\
\hline & Média & Erro Padrão & Desvio Padrão & Média & Erro Padrão & Desvio Padrão \\
\hline Vigor & 4,24 & 0,01 & 1,09 & 4,10 & 0,03 & 1,28 \\
\hline Dedicação & 4,33 & 0,01 & 1,36 & 4,25 & 0,04 & 1,42 \\
\hline Absorção & 3,77 & 0,01 & 1,28 & 4,06 & 0,03 & 1,27 \\
\hline Total & 4,10 & 0,01 & 1,11 & 4,13 & 0,03 & 1,33 \\
\hline
\end{tabular}

Nota. *Adaptado de Schaufeli e Bakker (2009, p. 30).

\section{Tabela 9}

Scores de Distribuição em Percentagens da UWES-17 e da UWES-17 no Presente Estudo

\begin{tabular}{|c|c|c|c|c|c|c|c|}
\hline \multicolumn{4}{|c|}{$\begin{array}{c}\text { UWES-17 } \\
(N=12161)\end{array}$} & \multicolumn{4}{|c|}{$\begin{array}{l}\text { UWES-17 no presente estudo } \\
\qquad(N=1369)\end{array}$} \\
\hline Vigor & Dedicação & Absorção & Score Total & Vigor & Dedicação & Absorção & Score Total \\
\hline 0,6 & 1,3 & 2,0 & 0,7 & 1,5 & 2,0 & 1,9 & 1,2 \\
\hline 2,5 & 3,9 & 6,6 & 3,5 & 6,1 & 6,8 & 5,8 & 6,4 \\
\hline 9,4 & 9,7 & 16,3 & 11,8 & 11,8 & 9,9 & 11,4 & 11,8 \\
\hline 22,1 & 18,0 & 25,2 & 24,9 & 27,2 & 23,0 & 31,4 & 19,2 \\
\hline 34,4 & 26,8 & 28,9 & 34,8 & 18,4 & 15,6 & 19,2 & 29,6 \\
\hline 31,1 & 40,3 & 21,0 & 24,3 & 35,0 & 42,7 & 30,3 & 31,6 \\
\hline
\end{tabular}

Nota. * Adaptado de Schaufeli e Bakker (2009, p. 30).

9 Schaufeli e Bakker (2009, p. 30) definiram os scores normativos de acordo com valores: de o a 0,99 (1 - "algumas vezes por ano"); de 1 a 1,99 (2 - “uma vez ou menos por mês"); de 2 a 2,99 (3 - “algumas vezes por mês"); de 3 a 3,99 (4 - "uma vez por semana"); de 4 a 4,99 (5 - “algumas vezes por semana"); e de 5 a 6 (6 - "todos os dias"). 
Tabela 10

Scores Normativos para o UWES-17 e a UWES-17 no Presente Estudo

\begin{tabular}{|c|c|c|c|c|c|c|c|c|}
\hline & \multicolumn{4}{|c|}{$\begin{array}{c}\text { UWES-17 } \\
(N=2313) *\end{array}$} & \multicolumn{4}{|c|}{$\begin{array}{l}\text { UWES-17 no presente estudo } \\
\qquad(N=1369)\end{array}$} \\
\hline & Vigor & Dedicação & Absorção & Score Total & Vigor & Dedicação & Absorção & Score Total \\
\hline Média & 3,99 & 3,81 & 3,56 & 3,82 & 4,60 & 4,72 & 4,51 & 4,65 \\
\hline Desvio padrão & 1,11 & 1,31 & 1,18 & 1,10 & 1,31 & 1,38 & 1,29 & 1,28 \\
\hline Erro padrão & 0,01 & 0,01 & 0,01 & 0,01 & 0,04 & 0,04 & 0,03 & 0,03 \\
\hline
\end{tabular}

Nota. * Adaptado de Schaufeli e Bakker (2009, p. 32).

Tabela 11

Categorização dos Scores da UWES-17

\begin{tabular}{|c|c|c|c|c|}
\hline & \multicolumn{4}{|c|}{$\begin{array}{l}\text { UWES-17 } \\
(N=2313)\end{array}$} \\
\hline & Vigor & Dedicação & Absorção & Score Total \\
\hline Muito Baixo & $\leq 2,17$ & $\leq 1,60$ & $\leq 1,60$ & $\leq 1,93$ \\
\hline Baixo & $2,18-3,20$ & $1,61-3,00$ & $1,61-2,75$ & $1,94-3,06$ \\
\hline Médio & $3,21-4,80$ & $3,01-4,90$ & $2,76-4,40$ & $3,07-4,66$ \\
\hline Alto & $4,81-5,65$ & $4,91-5,79$ & $4,41-5,35$ & $4,67-5,53$ \\
\hline Muito Alto & $\geq 5,61$ & $\geq 5,80$ & $\geq 5,36$ & $\geq 5,54$ \\
\hline
\end{tabular}

Nota. * Adaptado de Schaufeli e Bakker (2009, p. 32).

Tabela 12

Coeficientes de Correlação de Pearson da UWES-17 e UWES-17 Aplicado a Assistentes Sociais Portugueses

\begin{tabular}{|c|c|c|c|c|}
\hline & \multicolumn{3}{|c|}{$\begin{array}{c}\text { UWES-17 } \\
(N=12631) *\end{array}$} & \multirow{2}{*}{$\begin{array}{l}\text { UWES-17 no presente estudo } \\
\qquad(N=1369) \\
\text { Total }\end{array}$} \\
\hline & Total & Média & Intervalo & \\
\hline Vigor - Dedicação & 0,93 & 0,94 & $0,82-0,99$ & $0,86 * *$ \\
\hline Dedicação - Absorção & 0,85 & 0,91 & $0,75-0,94$ & $0,81 * *$ \\
\hline Vigor - Absorção & 0,86 & 0,90 & $0,73-0,99$ & $0,83^{* *}$ \\
\hline
\end{tabular}

Nota. *adaptado de Schaufeli e Bakker, 2009, p. 24; ** $p<0,01$.

Tendo por base os valores de referência fixados podemos afirmar que o score das variáveis "vigor" e "dedicação" da UWES-17 no presente estudo, assim como o seu score total, apresentam valores médios de 4,60, 4,72 e 4,65 respetivamente, integrando-se na categorização "médio". Destaca-se, no entanto, que a classificação da variável "absorção" é "alta” na medida em que o seu score apresenta o valor médio de 4,51.

A análise realizada aos scores vem, também, validar os anteriores testes, permitindo garantir que a UWES-17 apresenta características psicométricas consideradas muito boas em comparação com a UWES-17, pelo que se revela um instrumento adequado para aferir o nível de engagement dos assistentes sociais em Portugal.

No intuito de analisar as relações estabelecidas entre as dimensões da UWES, recorremos ao cálculo dos coeficientes de correlação de Pearson, cujos valores encontrados expressos na Tabela 12 denotam uma associação significativamente positiva, na medida em que as correlações entre as dimensões apresentam-se dentro dos intervalos estabelecidos pelos autores 
(Salanova et al., 2000; Schaufeli et al., 2002; Schaufeli, Martinez, Marques Pinto, Salanova e Bakker, 2002).

Dado que estatisticamente as correlações são significativas quando $p<0,01$, afere-se que as três dimensões da UWES-17 no nosso estudo estão intimamente correlacionadas entre si. A correlação mais elevada observa-se entre o "vigor" e a "dedicação" $(0,86)$ e a mais baixa entre a "dedicação" e a "absorção" $(0,81)$. A alta correlação entre as três dimensões (Tabela 10) e os valores adequados do Alfa de Cronbach, tanto para o total da escala como para as dimensões "vigor”, “dedicação" e "absorção" (Tabela 7) da UWES-17 no presente estudo.

\section{DISCUSSÃO E CONCLUSÕES}

Este estudo realizou-se com o objetivo de avaliar os itens e as respetivas dimensões da Utrecht Work Engagement Scale (UWES-17) de Schaufeli e Bakker (2009), aplicada a assistentes sociais a exercerem funções em Portugal.

Para tal foram analisados e comparados os valores do Alfa de Cronbach, média, erro e desvio padrão, bem como a distribuição e classificação dos scores da UWES-17 aplicada a assistentes sociais com a UWES-17. Relativamente ao Alfa de Cronbach (UWES-17 $=0,93$; UWES-17 no presente estudo $=0,952$ ) verificouse que o instrumento tem um elevado grau de fiabilidade. Quer as dimensões, quer os 17 itens que compõem a escala detêm uma consistência interna classificada como muito boa, de acordo com diferentes autores (Davis, 1964; Kaplan e Saccuzzo, 1982; Murphy e Davidsholder, 1988; Nunnally, 1978).

Quando analisadas os resultados das médias, erros e desvios padrão da UWES-17 do presente estudo e comparados com os da UWES-17, não foram identificados valores discrepantes significativos.

No que respeita à análise dos scores, também não foram identificadas discordâncias entre os dados obtidos na UWES-17 e na UWES-17 no presente estudo. Os scores das variáveis "vigor" e "dedicação", assim como do score total, apresentam valores dentro da média. O score da variável "absorção" classifica-se como elevado.

A análise fatorial exploratória confirmou o número de dimensões do estudo original, mas apresentou resultados diferentes no agrupamento dos itens de cada dimensão.

Parafraseando Siegerti et al. (1987), não podemos deixar de referir que a distribuição dos itens pelas dimensões pode variar consoante as populações. Mais, uma vez que todos os itens avaliam o engagement, pode acontecer alguma interpenetração entre os diferentes domínios. Talvez esta diferença também possa estar relacionada com os conteúdos dos itens. Por exemplo, o item 12 ("Sou capaz de ficar a trabalhar por períodos de tempo muito longos"), segundo os autores da escala pertence à dimensão vigor, no entanto, no nosso estudo, surge juntamente com dois itens da dimensão absorção, que parecem ter ligação de conteúdo (Item 14 - "Deixo-me ir quando estou a trabalhar" e Item 16 - "É-me difícil desligar do meu trabalho"). É ainda de referir, que segundo o Manual UWES (Schaufeli e Bakker, 2009) a alta correlação entre as três dimensões podem levar a que se considere um modelo unidimensional para esta escala. Aliás, tal como é possível confirmar no presente estudo as três dimensões da UWES-17 estão intimamente correlacionadas entre si.

Após o apuramento das suas características psicométricas afirma-se que a UWES-17 aplicada a assistentes sociais pode ser considerada um instrumento válido de medição do nível do engagement dos assistentes sociais a exercerem funções em Portugal.

Espera-se que a adaptação e validação desta escala possa estimular não só o interesse pela teorização acerca do envolvimento no trabalho, como também servir de ferramenta para a promoção do comportamento organizacional positivo e da prevenção do burnout no campo da intervenção social.

Conflito de interesses | Conflict of interest: nenhum | none.

Fontes de financiamento | Funding sources: nenhuma | none

\section{REFERÊNCIAS}

Abdallah, T. (2009). Prevalence and predictors of burnout among palestinian social workers. International Social Work, 52(2), 223-233. doi:10.1177/0020872808099732

Angst, R., Benevides-Pereira, A. e Porto-Martins, P. (2009). UWES manual - português BR. Rio de Janeiro: GEPEB - Grupo de Estudos e Pesquisas sobre Estresse e Burnout.

Aritzeta, A. e Gartzia, L. (2009). Inteligencia emocional y burnout en profesionales de la intervención social y comunitaria. Em P. Fernández Berrocal et al. (Ed.), Avances en el estudio de la inteligencia emocional - I congreso internacional de inteligencia emocional (pp. 173-178). Málaga: Fundación Marcelino Botín.

Bakker, A., Schaufeli, W., Leiter, M. e Taris, T. (2008). Work engagement: An emerging concept in occupational health psychology. Work and Stress, 22(3), 187-200. doi: $10.1080 / 02678370802393649$

Branco, F. (2009). A profissão de assistente social em Portugal. Locus SOCI@L, 3, 61-89. 
Carrera, J. (2011). Stress e burnout: Um estudo de caso de assistentes sociais que trabalham com idosos em IPSS's. [Dissertação de mestrado]. Recuperado em http://www.repository.utl.pt/ b i t s t r e a m/ $104000.5 / 3097 / 1 / \mathrm{T}$. PS\%20Joana\%2oFigueiredo\%20Carrera.pdf.

Collins, S. (2008). Statutory social workers: Stress, job satisfaction, coping, social support and individual differences. British Journal of Social Work, 38, 1173-1193. doi:10.1093/bjsw/bcmo47

Costello, A. e Osbourne, J. (2005). Best Practices in Exploratory Factor Analysis: four recommendations for getting the most from your analysis. Practical assessment, Research and Evaluation, 10(7), 1-9.

Csikszentmihalyi, M. (1990). Flow: The psychology of optimal experience. New York: Harper and Row.

Davies, R. (1998). Stress in social work. London: Jessica Kingsley Publishers.

Davis, F. (1964). Educational measurements and their interpretation. Belmont: Wadsworth Publishing Co.

Demerouti, E., Bakker, A., Janssen, P., e Schaufeli, W. (2001). Burnout and engagement at work as a function of demands and control. Scandinavian Journal of Work, Environment \& Health, 27, 279-286.

Durán, M. A., Extremera, N. e Rey, L. (2005). Burnout y engagement en el marco de la experiencia piloto para la adaptación a los créditos europeos. Em J. Romay Martínez e R. García Mira (Eds), Psicología Social y Problemas Sociales (pp. 741-749). Madrid: Biblioteca Nueva.

Extremera, N., Durán, M. e Rey, L. (2005). La inteligencia emocional percibida y su influencia sobre la satisfacción vital, la felicidad subjetiva y el "engagement" en trabajadores de centros para personas con discapacidad intelectual. Ansiedad y Estrés, 11, 63-73.

Fong, T. C. e Ng, S. (2012). Measuring engagement at work: Validation of the chinese version of the utrecht work engagement scale. International Journal of Behavioral Medicine, 19(3), 391-397. doi: 10.1007/s12529-011-9173-6

Hamama, L. (2012). Burnout in social workers treating children as related to demographic characteristics, work environment, and social support. Social Work Research, 36(2), 113-125. doi: 10.1093/ swr/svs003

Hill, M., e Hill, A. (2002). Investigação por questionário. Lisboa: Edições Sílabo.

Kahn, W. A. (1990). Psychological conditions of personal engagement and disengagement at work. Academy of Management Journal, 33(4), 692-724. doi:10.2307/256287

Kaplan, R., e Saccuzzo, D. (1982). Psychological testing: Principles, applications and issues. Monterey: Brooks/Cole Publishing Company.

Kim, H. J. (2011). Burnout and physical health among social workers: A three-year longitudinal study. Social Work, 56(3), 258-268. doi: $10.1093 / \mathrm{sw} / 56.3 .258$

Lloyd, C., King, R. e Chenoweth, L. (2002). Social work, stress and burnout: A review. Journal of Mental Health, 11(3), 255-265. doi: $10.1080 / 09638230020023642$

Lonne, B. (2003). Social workers and human service practitioners. Em M. Dollard, H. Winefield e A. Winefield (Eds), Occupational stress in the service professions (pp. 281-309). London e New York: Taylor e Francis Group.

Malheiro, J. (2010). A satisfação laboral e a confiança organizacional em três empresas de tecnologias da informação: um estudo exploratório. Em E. Vaz e V. Meirinhos (Coords.). Recursos
Humanos: das teorias às boas práticas. Porto: Editorial Novembro.

Martínez, I. M., e Salanova, M. (2003). Niveles de burnout y engagement en estudiantes universitarios: Relación con el desempeño y desarrollo profesional. Revista de Educación, 330, 361-384.

Martins, J. (2013). Validação da Versão Portuguesa do Questionário Utrecht Work Engagement para medir o engagement no trabalho nos profissionais dos cuidados de saúde primários [Dissertação de mestrado não publicada]. ISCTE, Lisboa.

Maslach, C. e Leiter, M. (1997). The truth about burnout: How organizations cause personal stress and what to do about it. San Francisco: CA: Jossey-Bass.

Montgomery, A., Peeters, M., Schaufeli, W. e Den Ouden, M. (2003). Work-home interference among newspaper managers: Its relationship with Burnout and engagement. Anxiety, Stress \& Coping, 16, 195-211. doi:10.1080/10615806.2003.10382973

Murphy, K. e Davidsholder, C. (1988). Psychological testing: Principles and applications. Englewood Cliffs, New Jersey: Prentice Hall.

Nunnally, J. (1978). Psychometric theory. New York: McGraw-Hill Inc.

Pestana, M. e Gageiro, J. (2000). Análise de dados para ciências sociais. Lisboa: Edições Sílabo.

Peterson, R. (1994). A meta-analysis of Cronbach's coefficient alpha. Journal of Consumer Research, 21 (2), 381-391. Recuperado em http://www.jstor.org/stable/2489828

Ramiro, B. (2014). Una aproximación a la influencia de la inteligencia emocional percibida en su relación con los niveles de burnout y engagement en el desempeño del trabajo social. Azarbe: Revista Internacional de Trabajo Social y Bienestar, 3, 123-131. Recuperado em http://revistas.um.es/azarbe/article/view/198451/161721

Salanova, M., Brescó, E. e Schaufeli, W. (2005). Hacia un modelo espiral de las creencias de eficacia en el estudio del burnout y del engagement. Ansiedad y Estrés, 11(2-3), 215-231.

Salanova, M., Carrero, V., Pinanzo, D. e Schaufeli, W. (2003). Job characteristics and proactive behaviour: The mediating role of job engagement. Journal of Occupational and Organizational Psychology, 17, 199-210.

Salanova, M., Grau, R., Llorens, S. e Schaufeli, W. (2001). Exposición a las tecnologías de la información, burnout y engagement: El rol modulador de la autoeficacia profesional. Psicología Social Aplicada, 11, 69-89.

Salanova, M., Schaufeli, W., Llorens, S., Peiró, J. e Grau, R. (2000). Desde el "bumout" al "engagement": Una nueva perspectiva. Revista de Psicología del Trabajo y de las Organizaciones, 16, 117-134.

Sarmento, M. (2008). Guia prático sobre a metodologia científica para a elaboração, escrita e apresentação de teses de doutoramento, dissertações de mestrado e trabalhos de investigação aplicada ( $2^{a}$ ed.). Lisboa: Universidade Lusíada Editora.

Schaufeli, W. e Bakker, A. (2003). UWES - Utrecht work engagement scale. Utrecht: Utrecht University - Occupational Health Psychology Unit.

Schaufeli, W. e Bakker, A. (2004). Job demands, job resources, and their relationship with burnout and engagement: A multi-sample study. Journal of Organizational Behavior, 25, 293-315. doi:10.1002/ job. 24

Schaufeli, W. e Bakker, A. (2009). UWES - escala de engagement no trabalho de utrecht. Maringá: GEPEB - Grupo de Estudos e Pesquisas sobre Estresse e Burnout. 
Schaufeli, W., Martinez, I., Marques Pinto, A., Salanova, M. e Bakker, A. (2002). Burnout and engagement in university students: A cross national study. Journal of Cross-Cultural Psychology, 33, 464-481. doi:10.1177/0022022102033005003

Schaufeli, W., Salanova, M., Gonzalez-Roma, V. e Bakker, A. (2002). The measurement of engagement and burnout and: A two sample confirmatory factor analytic approach. Journal of Happiness Studies, 3(1), 71-92. doi:10.1023/A:1015630930326
Siegertil, R., McCormick, I., Taylor, A. e Walkey, F. (1987). An examination of reported factor structures of the General Health Questionnaire and the identification of a stable replicable structure. Australian Journal of Psychology, 39(1), 89-100. doi: 10.1080/00049538708259038

Takeda, F., Yokoyama, E., Miyake, T. e Ohida, T. (2002). Mental health and job factors in social workers at social welfare offices. Journal Occupational Health, 44, 385-390. doi:10.1539/joh.44.385 